\title{
Genetic variations in triple-negative breast cancers undergoing neo-adjuvant chemotherapy
}

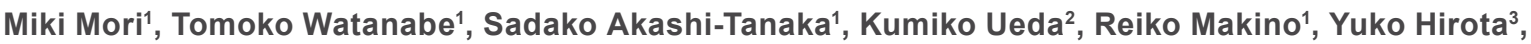 \\ Seigo Nakamura ${ }^{1}$
}

'Department of Breast Surgical Oncology, Showa University, Shinagawaku, Tokyo 142-8666, Japan.

${ }^{2}$ Clinical Research Laboratory, Showa University, Shinagawaku, Tokyo 142-8666, Japan.

${ }^{3}$ Department of Pathology, Showa University, Shinagawaku, Tokyo 142-8666, Japan.

Correspondence to: Dr. Miki Mori, Department of Breast Surgical Oncology, Showa University, 1-5-8 Hatanodai, Shinagawaku, Tokyo 142-8666, Japan. E-mail: wilsonmikity@yahoo.co.jp

\begin{abstract}
How to cite this article: Mori M, Watanabe T, Akashi-Tanaka S, Ueda K, Makino R, Hirota Y, Nakamura S. Genetic variations in triple-negative breast cancers undergoing neo-adjuvant chemotherapy. Cancer Drug Resist 2019;2:877-84.

http://dx.doi.org/10.20517/cdr.2019.44
\end{abstract}

Received: 29 May 2019 First Decision: 15 Jul 2019 Revised: 17 Jul 2019 Accepted: 29 Jul 2019 Published: 19 Sep 2019

Science Editor: Helen M. Coley Copy Editor: Cai-Hong Wang Production Editor: Tian Zhang

\begin{abstract}
Aim: Triple negative breast cancer (TNBC) is known as aggressive subtype and have no identified targeted therapies. We examined the relationship of neoadjuvant chemotherapy response to genetic variations of TNBC.

Methods: The tumors used in this study were collected from Showa University Hospital, Japan. Thirteen formalinfixed paraffin-embedded tumors from Japanese TNBC patients who underwent neoadjuvant chemotherapy were used for analysis. Of these, eight surgically resected tumors showed progressive disease and/or recurrence after treatment $(P D / R E C)$, and biopsy tissues from five patients showing pathological complete response ( $p C R$ ) were analyzed. DNA extracted from tissue sample were analyzed. The Miseq system and Trusight Tumor Sequence panel kit were used to sequence 174 amplicons over 82 exons of 26 cancer-related genes to identify genetic mutations.
\end{abstract}

Results: Seven somatic non-synonymous variants were detected in three genes (FOXL2, PIK3CA, and TP53) in all five pCR patients, and six somatic non-synonymous variants in two genes (PTEN and TP53) were detected in six of eight PD/ REC patients. Eight of 13 TNBC tumors were found to have TP53 pathogenic variants, in both pCR and PD/REC cases.

Conclusion: Although TP53 variation was detected in both PCR and PD/REC cases, each location and type of the variant were different. We could not identify genetic mutations associated with chemotherapy response and recurrence.

Keywords: Triple negative breast cancer, next generation sequence, TP53 and neo adjuvant chemotherapy

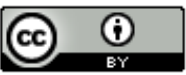

(C) The Author(s) 2019. Open Access This article is licensed under a Creative Commons Attribution 4.0 International License (https://creativecommons.org/licenses/by/4.0/), which permits unrestricted use sharing, adaptation, distribution and reproduction in any medium or format, for any purpose, even commercially, as long as you give appropriate credit to the original author(s) and the source, provide a link to the Creative Commons license, and indicate if changes were made. 


\section{INTRODUCTION}

Triple-negative breast cancer (TNBC) is defined as any breast cancer lacking estrogen receptor, progesterone receptor, and human epidermal growth factor receptor 2 expression. It accounts for approximately $15 \%-20 \%$ of all breast cancers ${ }^{[1,2]}$. TNBC is reported to behave more aggressively and is associated with a worse survival than other types of breast cancer ${ }^{[3,4]}$. Patients with TNBC have no identified targeted therapies. They are currently treated by surgery and chemotherapy alone ${ }^{[1]}$.

Triple-negative subtypes are more sensitive to neo-adjuvant chemotherapy than luminal breast cancers ${ }^{[5-7]}$. Patients with TNBC have increased pathologic complete response (pCR) rates compared with nonTNBC patients, and those with pCR have excellent survival regardless of subtype $e^{[5-7]}$. However, patients with progressive disease after neo-adjuvant chemotherapy have a significantly worse prognosis if they have TNBC compared with other subtypes ${ }^{[5,7]}$. About $70 \%-80 \%$ of patients of TNBC fail to respond to neoadjuvant chemotherapy $(\mathrm{NAC})^{[5-7]}$, but the molecular mechanism underlying differences in responders and non-responders is unclear. It is also poorly understood which genes and pathways are related to treatment response in breast tumors.

Herein, we performed an exploratory study using next generation sequencing to investigate the dynamics of tumor gene mutations in TNBC patients receiving NAC.

\section{METHODS}

\section{Ethical approval}

This study was approved (number 1589) by the Institutional Review Board of Showa University, Japan. Written informed consent was received from the patients. If informed consent could not be received for the death of patients, the candidate opted out on the homepage of the hospital.

\section{Patient information}

The tumors analyzed in this study were collected from Showa University Hospital, Japan. Ninety four patients revealed triple negative subtype of 1111 patients of primary breast cancer surgery between 2011 and 2013. Of these 94 patients, 33 underwent neo adjuvant chemotherapy prior to surgery. Ten patients achieved pCR and remaining 23 patients had residual disease. Four patients revealed clinically progressive disease during neo adjuvant chemotherapy. Taxane followed by anthracycline based regimens were applied as standard neo-adjuvant chemotherapy. Two patients who revealed progressive disease during first taxane regimen dropped out of neo-adjuvant chemotherapy then planned surgery without following anthracycline chemotherapy. Seven patients of 23 patients who had residual disease including 3 progressive disease revealed recurrence in the same period. In total, there were 8 patients with progressive disease and/or recurrence (PD/REC). Five of $10 \mathrm{pCR}$ cases underwent pretreatment biopsy in Showa University Hospital and able to obtain the sample. These $8 \mathrm{PD} / \mathrm{REC}$ cases and $5 \mathrm{pCR}$ cases were analyzed. Formalinfixed paraffin-embedded (FFPE) samples from pretreatment biopsy of PD/REC and pCR cases and surgical specimen of PD/REC cases were obtained. Clinical and pathologic information was obtained from medical chart including the information of the results of both germline BRCA1/2 testing and BRNAness. The concept of "BRCAness" is that sporadic basal-like breast cancers resemble BRCA1 mutated cancers. A set of 34 MLPA probes was used to identify BRCAness ${ }^{[8]}$. American Joint Committee on Cancer/tumor-nodemetastasis cancer staging was assessed.

\section{Preparation of genomic DNA}

Ten slices of $10 \mu \mathrm{m}$-thick FFPE tissues were used for genomic DNA extraction. Tissues were de-paraffinized with xylene, then DNA was extracted using the QIAamPD/recNA FFPE Tissue Kit (Qiagen, Venlo, Netherlands) following the manufacturer's instructions. 
Briefly, each deparaffinization solution, specific buffers and proteinase was added to destruct cells while centrifuging. After incubation, ethanol was added to precipitate DNA. After transferring the entire lysate to purification column, the centrifugation was repeated. Finally, elution buffer was added to elute DNA in the column.

\section{Target genes}

The TruSight Tumor Sequencing panel (Illumina, San Diego, California, USA, \#FC-130-2001) was used to detect genetic variations in tumors using NGS. This panel covered 174 amplicons over 82 exons of 26 cancer-related genes. The following genes encode kinases: AKT1, ALK, BRAF, EGFR, ERBB2, FGFR2, KIT, MAP2K1, MET, PD/RECGFRA, PIK3CA, SRC, and STK11, while the remainder do not: APC, CDH1, CTNNB1, FBXW7, FOXL2, GNAQ, GNAS, KRAS, MSH6, NRAS, PTEN, SMAD4, and TP53.

\section{Sequencing}

The quality of the genomic DNA purified from FFPE tissues was checked by quantitative PCR using Quality Control Template (Illumina) and $\mathrm{iQ}^{\mathrm{TM}} \mathrm{SYBR}^{\oplus}$ Green Supermix (BioRad). Samples were required to fulfill the criteria $\Delta \mathrm{Ct}<5$, which was typically 30-300 ng depending on the DNA quality. However, $\Delta \mathrm{Ct}$ = 12 biopsy specimens were also included in the analysis. Genomic DNA hybridization-based enrichment was performed using the TruSight Tumor Sequence panel kit according to the manufacturer's protocol. Pooled amplicons were end-repaired then underwent adapter ligation. The purified library was quantified using the Qubit ${ }^{\circledR}$ fluorometer with the Qubit ${ }^{\circledR}$ dsDNA BR assay kit. Finally, the captured DNA was subjected to 121-bp paired-end read sequencing on the MiSeq system (Illumina).

\section{Strategies for the analysis of genetic variations}

Sequence reads were exported into FASTQ format files. The alignment of pair-end sequencing reads to the reference genome was performed using a banded Smith-Waterman method with the AmpliconDS workflow software plug-in for MiSeq Reporter. Human Genome version 19 (UCSC) was used as the alignment reference. The identification of sequencing variants was performed using the Illumina Somatic Variant Caller algorithm. The called SNPs/indels were annotated using COSMIC (somatic mutation information), the IARC TP53 database (human TP53 gene variations related to cancer) ${ }^{[9]}$, and other databases of genetic variations. Database was aggregated into the Illumina VariantStudio application. Additionally, NM_000179.2:C.3254delC and NM_000179.2:C.3253_3254insC in MSH6 were eliminated as known artifact variants. Statistical analysis was performed with Fisher's exact test.

\section{RESULTS}

\section{Clinical features of the patients}

The clinical features of the patients are summarized in the Table 1. Patients had an average age of 49.6 years. Seven cases underwent $B R C A 1$ and $B R C A 2$ germline mutation testing. BRCA1/2 gene testing are not covered by national healthcare insurance, so it was performed to limited patients based on age, family history by self-pay. Seven PD/REC cases, including three patients with germline mutation showed BRCAness. One pCR case also showed BRCAness.

\section{Genetic variations in tumors showed PD/REC and $\mathrm{PCR}$}

The genetic variations after filtering out synonymous variants and known artifacts are shown in the Table 2 . Three biopsy samples of $8 \mathrm{PD} / \mathrm{REC}$ cases were not available for the analysis. In PD/REC cases, we found ten non-synonymous variants were detected in the coding regions of two genes in 5 biopsy tissues before NAC, while 14 non-synonymous variants were detected in the coding regions of three genes in 8 surgical specimens after NAC. In 5 pCR cases, ten non-synonymous variants were detected in the coding regions of four genes. Of these, variants TP53 Val31Ile and Pro72Arg, STK11 Phe354Leu, and MET Asn375Ser are known polymorphisms (NIH dbSNP: rs33917957, rs1042522, rs59912467 and rs33917957, respectively). 
Table 1. Clinical features of the patients

\begin{tabular}{|c|c|c|}
\hline & pCR & PD/REC \\
\hline \multicolumn{3}{|l|}{ Age, year } \\
\hline Median (range) & $56(40-72)$ & $46(27-68)$ \\
\hline \multicolumn{3}{|l|}{ Clinical T-stage at diagnosis } \\
\hline $\mathrm{T} 1$ & 1 & 0 \\
\hline $\mathrm{T} 2$ & 2 & 7 \\
\hline T3 & 2 & 1 \\
\hline $\mathrm{T} 4$ & 0 & 0 \\
\hline \multicolumn{3}{|l|}{ Clinical N-stage at diagnosis } \\
\hline NO & 2 & 5 \\
\hline N1 & 1 & 3 \\
\hline N2 & 2 & 0 \\
\hline N3 & 0 & 0 \\
\hline \multicolumn{3}{|l|}{ Stage at diagnosis } \\
\hline 1 & 1 & 0 \\
\hline II & 1 & 7 \\
\hline III & 3 & 1 \\
\hline IV & 0 & 0 \\
\hline \multicolumn{3}{|l|}{ Nuclear grade } \\
\hline 1 & 0 & 0 \\
\hline 2 & 1 & 3 \\
\hline 3 & 4 & 4 \\
\hline Not available & 0 & 1 \\
\hline \multicolumn{3}{|l|}{ Ki-67 } \\
\hline$<20 \%$ & 0 & 0 \\
\hline$\geq 20 \%$ & 5 & 7 \\
\hline Not available & 0 & 1 \\
\hline \multicolumn{3}{|l|}{ Histology } \\
\hline Ductal & 4 & 8 \\
\hline Lobular & 0 & 0 \\
\hline Others & 1 & 0 \\
\hline \multicolumn{3}{|l|}{ Subtype defined by IHC } \\
\hline $\operatorname{EGFR}(+), \mathrm{CK} 5 / 6(+)$ & 0 & 2 \\
\hline $\operatorname{EGFR}(+)$, CK5/6(-) & 0 & 1 \\
\hline EGFR(-), CK5/6(+) & 1 & 1 \\
\hline EGFR(-), CK5/6(-) & 2 & 3 \\
\hline Not tested & 2 & 1 \\
\hline \multicolumn{3}{|l|}{ BRCA mutation } \\
\hline Deleterious & 0 & 3 \\
\hline Negative & 2 & 2 \\
\hline Not tested & 3 & 3 \\
\hline \multicolumn{3}{|l|}{ BRCAness } \\
\hline Yes & 1 & 7 \\
\hline No & 1 & 0 \\
\hline Not analyzed & 3 & 1 \\
\hline \multicolumn{3}{|l|}{ Neo-adjuvant treatment } \\
\hline Anthracycline + Taxane & 4 & 6 \\
\hline Taxane + Other & 0 & 2 \\
\hline Other & 1 & 0 \\
\hline \multicolumn{3}{|l|}{ Surgery type } \\
\hline Mastectomy & 1 & 6 \\
\hline Lumpectomy & 4 & 2 \\
\hline Sentinel Node Biopsy only & 2 & 4 \\
\hline Axillary lymph node dissection & 3 & 4 \\
\hline \multicolumn{3}{|l|}{ Yeild of pathological T-stage } \\
\hline TO & 5 & 0 \\
\hline T1 & 0 & 4 \\
\hline $\mathrm{T} 2$ & 0 & 3 \\
\hline T3 & 0 & 1 \\
\hline
\end{tabular}




\begin{tabular}{|c|c|c|}
\hline T4 & 0 & 0 \\
\hline \multicolumn{3}{|l|}{ Yeild of pathological N-stage } \\
\hline NO & 5 & 4 \\
\hline N1 & 0 & 4 \\
\hline N2 & 0 & 0 \\
\hline N3 & 0 & 0 \\
\hline \multicolumn{3}{|l|}{ Yeild of pathological stage } \\
\hline 1 & 0 & 3 \\
\hline II & 0 & 4 \\
\hline III & 0 & 1 \\
\hline IV & 0 & 0 \\
\hline \multicolumn{3}{|l|}{ Adjuvant treatment } \\
\hline No & 5 & 4 \\
\hline FEC & 0 & 1 \\
\hline Gemcitabine + Carboplatin & 0 & 3 \\
\hline \multicolumn{3}{|l|}{ Recurrence } \\
\hline Yes & 0 & 7 \\
\hline No & 5 & 1 \\
\hline \multicolumn{3}{|l|}{ Radiation } \\
\hline Yes & 4 & 4 \\
\hline No & 1 & 4 \\
\hline \multicolumn{3}{|l|}{ Death } \\
\hline Yes & 0 & 3 \\
\hline No & 5 & 5 \\
\hline Average DFS (months) & 25 & 17 \\
\hline Average observation period (months) & 34 & 40 \\
\hline
\end{tabular}

PCR: pathological complete response; PD/REC: progressive disease and/or recurrence; IHC: immunohistochemistry; DFS: disease free survival; FEC: fluorouracil epirubicin cyclophosphamide

As a result, total of 3 somatic mutations in one gene (TP53) and six somatic mutations in two genes (TP53 and PTEN) were detected in biopsy samples (3/5 cases) and surgical specimens of PD/REC patients (6/8 cases), respectively. Somatic mutations were detected in three genes TP53, FOXL2 and PIK3CA in pCR patients (5/5 cases).

TP53 was the most frequently mutated gene (9 of $13,69.2 \%$ in total, 5 of $8,62.5 \%$ in PD/REC cases and 4 of $5,80 \%$ in pCR cases $(P=0.50)$. Other pathogenic variants detected at a lower frequency were in genes PIK3CA $(n=1), P T E N(n=1)$ and FOXL2 $(n=1)$. In both PD/REC and CR, unique pathogenic variants were detected in TP53 and other genes. As shown in the Table 3, the TP53 Pro72Arg polymorphism was detected in both $\mathrm{PCR}$ and PD/REC cases.

Next, we compared the effect of chemotherapy in five matched cases showed PD/REC. In patient 7, the PTEN Ser179Valfs ${ }^{*} 8$ mutation was detected after NAC, but it was seen before NAC at low frequency on one strand (2\%). Other genetic variations (TP53 and STK11) were observed both pre- and post-treatment.

\section{DISCUSSION}

In this study, we used NGS to analyze 26 genes of 13 TNBC tumors receiving NAC. This is the first report to determine genetic variations in Japanese TNBC patients who showed pCR and PD/REC as a result of NAC. TP53 pathogenic variants were detected in both $\mathrm{pCR}$ and PD/REC cases at frequencies similar to the high rates previously reported. TP53 genetic variations in PCR cases might be associated with sensitivity to chemotherapy, and other TP53 variants in PD/REC cases might affect resistance to chemotherapy. Thus, sensitivity to chemotherapy may vary by the location and type of somatic TP53 variants. However, we could not confirm whether these genetic alterations found in this study were responsible for the chemosensitivity and recurrence because the number of patients are too small. 
Table 2. Genetic vatiations detected in PCR/PD groups

\begin{tabular}{|c|c|c|c|c|c|c|}
\hline \multirow{2}{*}{ Sample ID } & \multicolumn{3}{|c|}{ Biopsy samples before NAC } & \multicolumn{3}{|c|}{ Surgical specimens after NAC } \\
\hline & Gene & Pathological variations & Polymorphisms & Gene & Pathological variations & Polymorphisms \\
\hline \multicolumn{7}{|c|}{ Genetic variations in PD/REC patients } \\
\hline \multirow[t]{3}{*}{6} & TP53 & & p.Val31lle & TP53 & & p.Val31lle \\
\hline & TP53 & & p.Pro72Arg & TP53 & & p.Pro72Arg \\
\hline & STK11 & & p.Phe354Leu & STK11 & & p.Phe354Leu \\
\hline \multirow[t]{2}{*}{7} & TP53 & & p.Pro72Arg & TP53 & & p.Pro72Arg \\
\hline & & & & PTEN & p.Ser179Valfs ${ }^{\star} 8$ & \\
\hline 8 & TP53 & p.Val272Met & p.Pro72Arg & TP53 & p.Val272Met & p.Pro72Arg \\
\hline 9 & n.a. & & & TP53 & p.Arg333Valfs*12 & \\
\hline 10 & n.a. & & & TP53 & & p.Pro72Arg \\
\hline 11 & TP53 & p.Cys $275 \operatorname{Trp}$ & p.Pro72Arg & TP53 & p.Cys $275 \operatorname{Trp}$ & p.Pro72Arg \\
\hline 12 & n.a. & & & TP53 & p.Val216Met & \\
\hline 13 & TP53 & p.lle195Thr & p.Pro72Arg & TP53 & p.lle195Thr & p.Pro72Arg \\
\hline \multicolumn{7}{|c|}{ Genetic variations in $p C R$ patients } \\
\hline 1 & TP53 & p.Leu111Phefs ${ }^{*} 40$ & & & & \\
\hline \multirow[t]{2}{*}{2} & TP53 & p.Val157Phe & p.Pro72Arg & & & \\
\hline & Met & & p.Asn375Ser & & & \\
\hline 3 & TP53 & p.Cys141Tyr & p.Pro72Arg & & & \\
\hline \multirow[t]{2}{*}{4} & TP53 & p.Arg342* & p.Pro72Arg & & & \\
\hline & FOXL2 & p.Leu130GIn & & & & \\
\hline 5 & PIK3CA & p.Arg1023GInfs ${ }^{\star} 4$ & & & & \\
\hline
\end{tabular}

NAC: neoadjuvant chemotherapy; pCR: pathological complete response; PD/REC: progressive disease and/or recurrence; n.a.: not analyzed

The numbers of genes and cases assessed were limited in this study. However, the frequency of genetic variations detected in TNBC did not differ greatly from those in previous studies. Lips et al. ${ }^{[10]}$ analyzed 1977 genes in 56 pre-treatment TNBC biopsies of NAC responders and non-responders as well as matched normal DNA. They reported that most mutations were in TP53, TTN, and PIK3CA (55\%, 14\%, and 9\%, respectively). No recurrent mutations were associated with chemotherapy response or relapse ${ }^{[10]}$. Balko et al. ${ }^{[11]}$ examined residual disease in 74 clinically defined TNBCs after NAC, which included NGS performed on 20 matched pretreatment biopsies. Targeted NGS was carried out of 3320 exons from 182 oncogenes and tumor suppressors as well as 37 introns of 14 genes frequently rearranged in cancer. The frequency of each mutation was fewer than 5\% of samples. Alterations in TP53 were identified in 72 of 81 samples (89\%), which was a similar rate to that observed in other studies of basal-like breast cancer or TNBC, and The Cancer Genome Atlas dataset $(\sim 85 \%)^{[11]}$. Roy-Chowdhuri et al ${ }^{[12]}$ reported that a triple-negative group $(n$ $=77$ ) showed mutations in 15 of 46 genes tested, with TP53 showing the highest mutation frequency ( $n$ $=48 / 77,62 \%)$, followed by PIK3CA ( $n=13 / 77,17 \%), A P C, R E T, S M A D 4(n=2,3 \%), A K T 1, A T M, B R A F$, FGFR1, HRAS, JAK3, MET, SRC, PTEN, and STK11 $(n=1,1 \%)^{[12]}$. From these previous reports, it is difficult to conclude that the varied and sparse genetic alterations seen in the present study caused differences between responders and non-responders toward chemotherapy.

TP53 mutations in breast cancer have previously been reported to be associated with worse prognosis ${ }^{[13,14]}$. Ooe et al ${ }^{[15]}$ investigated the relationship between the p53 mutation status and response to docetaxel in breast cancers. They found that the response rate of patients with p53-mutated tumors (44\%) was lower than that of those with wild-type tumors (62\%). In addition, the TP53 p.Pro72Arg polymorphism was reported association with platinum-based chemotherapy response in non-small cell lung cancer ${ }^{[16]}$. Platinum-based chemotherapy was only administered to one case in our study, so no response difference was detected between heterozygous and homozygous variants in the $\mathrm{pCR}$ or PD/REC group. However, the same genetic variations of TP53 (p.V157F and p.Cys141Tyr) detected in PD/REC samples in previous study ${ }^{[11]}$ were also observed in pCR samples in our study. 
Table 3. Genotype of the TP53 Pro72Arg polymorphism

\begin{tabular}{ccclc}
\hline Sample ID & Genotype & Amino acid & Genotype & Respose to chemothrapy \\
\hline 1 & $\mathrm{C} / \mathrm{C}$ & Pro/Pro & - & $\mathrm{PCR}$ \\
2 & $\mathrm{C} / \mathrm{G}$ & Pro/Arg & Heterozygote & $\mathrm{PCR}$ \\
3 & $\mathrm{G} / \mathrm{G}$ & Arg/Arg & Homozygote & $\mathrm{PCR}$ \\
4 & $\mathrm{G} / \mathrm{G}$ & Arg/Arg & Homozygote & $\mathrm{PCR}$ \\
5 & $\mathrm{C} / \mathrm{C}$ & Pro/Pro & - & $\mathrm{PCR}$ \\
6 & $\mathrm{C} / \mathrm{G}$ & Pro/Arg & Heterozygote & $\mathrm{PD} / \mathrm{REC}$ \\
7 & $\mathrm{C} / \mathrm{G}$ & Pro/Arg & Heterozygote & PD/REC \\
8 & $\mathrm{G} / \mathrm{G}$ & Arg/Arg & Homozygote & $\mathrm{PD} / \mathrm{REC}$ \\
9 & $\mathrm{C} / \mathrm{C}$ & Pro/Pro & - & $\mathrm{PD} / \mathrm{REC}$ \\
10 & $\mathrm{C} / \mathrm{G}$ & Pro/Arg & Heterozygote & $\mathrm{PD} / \mathrm{REC}$ \\
11 & $\mathrm{G} / \mathrm{G}$ & Arg/Arg & Homozygote & $\mathrm{PD} / \mathrm{REC}$ \\
12 & $\mathrm{C} / \mathrm{C}$ & Pro/Pro & - & $\mathrm{PD} / \mathrm{REC}$ \\
13 & $\mathrm{C} / \mathrm{C}$ & Pro/Pro & - & $\mathrm{PD} / \mathrm{REC}$ \\
\hline
\end{tabular}

pCR: pathological complete response; PD/REC: progressive disease and/or recurrence

As a cohort, it is representative of Japanese TNBC, but the number of patients and samples was too low to confirm relevant relationship with genetic variants. This preliminary study should be followed up with a higher number of patients and selected genes in future.

In this study, 8 of 13 TNBC tumors were found to have TP53 pathogenic variants, in both pCR and PD/ REC cases. TP53 variations were detected at similarly high rates as previously reported and may be associated with sensitivity or resistance to chemotherapy depending on the location and type of the variant. If the effect of these genetic variations on the induction of apoptosis could be determined, this might enable the mechanism of the NAC chemotherapy response to be understood. Our results provide insights into potentially actionable variants for targeted therapeutic options in TNBC.

\section{DECLARATIONS}

\section{Authors' contributions}

Drafting of the manuscript, review and revisions of the final draft, data analysis and experiments: Mori M, Watanabe T, Makino R

Experiments: Ueda K, Hirota Y

Supervision, manuscript review and project administration: Akashi-Tanaka S, Nakamura S

\section{Availability of data and materials}

Not applicable.

\section{Financial support and sponsorship}

This work was supported in part by a Grant-in-Aid for the High-Technology Research Center Project from the Ministry of Education, Culture, Sports, Science and Technology of Japan.

\section{Conflicts of interest}

All authors declared that there are no conflicts of interest.

\section{Ethical approval and consent to participate}

This study was approved (number 1589) by the Institutional Review Board of Showa University, Japan. Written informed consent was received from the patients. If informed consent could not be received for the death of patients, the candidate opted out on the homepage of the hospital. 


\section{Consent for publication}

Not applicable.

\section{Copyright}

(c) The Author(s) 2019.

\section{REFERENCES}

1. Iwase H, Kurebayashi J, Tsuda H, Ohta T, Kurosumi M, et al. Clinicopathological analyses of triple negative breast cancer using surveillance data from the registration committee of the Japanese Breast Cancer Society. Breast Cancer 2010;17:118-24.

2. Foulkes WD, Smith IE, Reis-Filho. Triple-negative breast cancer. N Engl J Med 2010;363:1938.

3. Dent R, Trudeau M, Pritchard KI, Hanna WM, Kahn HK, et al. Triple-negative breast cancer: clinical features and patterns of recurrence. Clin Cancer Res 2007;13:4429-34.

4. Lin NU, Vanderplas A, Hughes ME, Theriault RL, Edge SB, et al. Clinicopathologic features, patterns of recurrence, and survival among women with triple-negative breast cancer in the National Comprehensive Cancer Network. Cancer 2012;118: 5463-72.

5. Luangdilok S, Samarnthai N, Korphaisarn K. Association between pathological complete response and outcome following neoadjuvant chemotherapy in locally advanced breast cancer patients. J Breast Cancer 2014;17:376-85.

6. Liedtke C, Mazouni C, Hess KR, André F, Tordai A, et al. Response to neoadjuvant therapy and long-term survival in patients with triple-negative breast cancer. J Clin Oncol 2008;26:1275-81.

7. Carey LA, Dees EC, Sawyer L, Gatti L, Moore DT, et al. The triple negative paradox: primary tumor chemosensitivity of breast cancer subtypes. Clin Cancer Res 2007;13:2329-34.

8. Lips EH, Laddach N, Savola SP, Vollebergh MA, Oonk AM, et al. Quantitative copy number analysis by Multiplex Ligation-dependent Probe Amplification (MLPA) of BRCA1-associated breast cancer regions identifies BRCAness. Breast Cancer Res 2011;13:R107.

9. Petitjean A, Mathe E, Kato S, Ishioka C, Tavtigian SV, et al. Impact of mutant p53 functional properties on TP53 mutation patterns and tumor phenotype: lessons from recent developments in the IARC TP53 database. Hum Mutat 2007;28:622-9.

10. Lips EH, Michaut M, Hoogstraat M, Mulder L, Besselink NJ, et al. Next generation sequencing of triple negative breast cancer to find predictors for chemotherapy response. Breast Cancer Res 2015;17:134.

11. Balko JM, Giltnane JM, Wang K, Schwarz LJ, Young CD, et al. Molecular profiling of the residual disease of triple-negative breast cancers after neoadjuvant chemotherapy identifies actionable therapeutic targets. Cancer Discov 2014;4:232-45.

12. Roy-Chowdhuri S, de Melo Gagliato D, Routbort MJ, Patel KP, Singh RR, et al. Multigene clinical mutational profiling of breast carcinoma using next-generation sequencing. Am J Clin Pathol 2015;144:713-21.

13. Olivier M, Langerød A, Carrieri P, Bergh J, Klaar S, et al. The clinical value of TP53 gene mutations in 1794 patients with breast cancer. Clin Cancer Res 2006;12:1157-67.

14. Langerød A, Zhao H, Borgan Ø, Nesland JM, Bukholm IR, et al. TP53 mutation status and gene expression profiles are powerful powerful prognositic markers of breast cancer. Breast Cancer Res 2007;9:R30.

15. Ooe A, Kato K, Noguchi S. Possible involvement of CCT5, RGS3, and YKT6 genes up-regulated in p53-mutated tumors in resistance to docetaxel in human breast cancers. Breast Cancer Res Treat 2007;101:305-15.

16. Shiraishi K, Kohno T, Tanai C, Goto Y, Kuchiba A, et al. Association of DNA repair gene polymorphisms with response to platinumbased doublet chemotherapy in patients with non-small-cell lung cancer. J Clin Oncol 2010;28:4945-52. 